\title{
Serum miR-148a And miR-152 Expression As Novel Noninvasive Biomarkers For Diagnosis And Prognostic Prediction of Multiple Myeloma
}

\author{
Lingling Xie ( $\nabla$ xll19882021@163.com ) \\ Affiliated Hospital of Nantong University https://orcid.org/0000-0002-0704-3551 \\ Guangfei Xie \\ Nantong University Affiliated Hospital: Affiliated Hospital of Nantong University \\ Xiuying Shi \\ Nantong University Affiliated Hospital: Affiliated Hospital of Nantong University \\ Hongming Huang \\ Nantong University Affiliated Hospital: Affiliated Hospital of Nantong University \\ Shaoqing Ju \\ Nantong University Affiliated Hospital: Affiliated Hospital of Nantong University \\ Xudong Wang \\ Nantong University Affiliated Hospital: Affiliated Hospital of Nantong University
}

\section{Research}

Keywords: Multiple myeloma, microRNAs, quantitative real-time polymerase chain reaction

Posted Date: September 7th, 2021

DOI: https://doi.org/10.21203/rs.3.rs-840039/v1

License: (9) This work is licensed under a Creative Commons Attribution 4.0 International License. Read Full License 


\section{Abstract}

Objective: Multiple myeloma (MM) as a hematological malignancy remains mostly incurable at present. The aim of the current study was to investigate the potential of serum microRNA (miRNA) as a novel biomarker for MM.

Methods: This study recruited $90 \mathrm{MM}$ patients and 30 healthy controls. Bone marrow samples were obtained from $12 \mathrm{MM}$ patients and 6 healthy controls. The expression level of miR-148a/152 was determined by qRT-PCR. The diagnostic performance and prognostic prediction of miR-148a/152 expression were analyzed by ROC curve and Kaplan-Meier method respectively. Serum IgA, IgG, $\mathrm{K}$ light chain, $\lambda$ light chain and $\beta_{2}$ microglobulin levels were detected by immunoturbidimetry. Serum LDH level was detected by lactic acid substrate method.

Results: The expression levels of miR-148a and miR-152 were elevated in serum and CD138+ plasma cells of MM patients as compared with controls $(P<0.05)$. There was a statistically significant correlation between serum miR-148a/152 expression and the clinicopathological parameters of MM patients.

Conclusion: The results of the present study suggest that circulating miR-148a and miR-152 may prove to be a marker for diagnosis and prognostic prediction of MM.

\section{Introduction}

Multiple myeloma (MM) is the second most common hematologic cancer with a global incidence of 6-7 cases per 100000 persons per year. It is characterized by abnormal proliferation of bone marrow (BM) plasma cells resulting in various clinical symptoms including anemia, hypercalcemia, renal insufficiency and osteolytic lesions [1]. Various laboratory tests including bone-marrow biopsy, determination of serum and urine free light chain, and low-dose whole-body CT, supportive MRI are useful for screening of MM [2]. But clinically, there is still a lack of novel noninvasive, specific and sensitive serum or plasma biomarkers for early diagnosis and follow up survey of MM.

MicroRNAs (miRNAs) as small non-coding RNA molecules that function in RNA silencing and post-transcriptional regulation of gene expression have gained popularity as potential biomarkers [3-5]. Aberrant expression of cellular miRNAs can lead to a variety of cancers; dysregulation of these miRNAs can have significant ramifications on tumor initiation, progression, and metastasis, and are often associated with diagnosis, prognosis and response to therapy. Circulating miRNAs are reported to be associated with tumor initiation, metastasis and chemotherapy resistance by regulating cancer stem cells. miRNA-21 is one of the important miRNAs implicated in the genesis and progression of human cancer. Up-regulation of cellular miRNA-21 can cause tumor development and progression, and circulating miRNA-21 has been described as a biomarker for different tumor entities. Plasma miRNA-21 can be used to differentiate early stage lung cancer patients from healthy non-smoking individuals [6]. Thus, given the characteristics of miRNAs in cell free components of the serum and a variety of other bodily fluids, circulating miRNA profiling can be more promising as biomarkers than earlier reported ones [7-9]. The circulating miRNA profile was reported to have a valuable role in early diagnosis, prognostic prediction, therapeutic decision-making and recurrence monitoring of MM [10-12]. A recent study demonstrated that miRNA-130a could discriminate between myeloma patients with extramedullary disease and healthy donors, offering a sensitivity of $77.1 \%$ and a specificity of $90.0 \%$ [13]. A combination of miR-720 and miR-1308 provides a powerful diagnostic tool for distinguishing normal healthy controls and patients with unrelated illnesses from patients with monoclonal gammopathy of undetermined significance (MGUS) and myeloma [14]. Previous studies reported the aberrant miRNA expression profile in CD138 + plasma cells from MM patients or MGUS, MM cell lines, the BM extracellular microenvironment, and serum or plasma of myeloma patients [10, 15-17].

miR-148a plays a role in several biological processes, including cellular differentiation and development. Differential expression of miR-148a can be observed in different cancers including gastric, colorectal, pancreatic, liver, esophageal, breast, non-small cell lung and urogenital system cancers. The expression level of miR-148a has been linked to the clinical classification, efficacy and prognosis of the tumor. miR-148a can act on different target genes via different pathways such as SMAD2 (TGF- $\beta$ /SMAD2 pathway), HPIP (AKT/ERK/FOXO4/ATFS pathway, mTOR pathway) and MET (HGF/Met/Snail pathway) to affect their functions in various tumors [18]. miR-152 is a member of the miR-148/152 family, which includes miR-148a, miR-148b and miR-152. miR-152 has been implicated in a variety of cancers such as prostate, pancreatic and gastric cancer and glioma. miR-152 was found to be inhibited in gynecological tumors such as ovary, endometrial and breast cancers, while its overexpression was found to be associated with 
resistance to cisplatin in ovarian cancer by inhibiting cell proliferation and promoting cell apoptosis via direct suppression of DNMT1 [19]. Thus, the miR-148/152 family plays a predominant role in the progression and chemosensitivity of various cancers. In spite of myriad research on these miRNAs, few studies have investigated their significance in MM. The aim of the present study was to assess the miR-148/miR-152 as potential biomarkers in serum and CD138 + plasma cells of MM patients.

\section{Materials And Methods Patients and samples}

Based on the guidelines for diagnosis and management of multiple myeloma in China (revised in 2011), a total of 90 newly diagnosed samples from clinically confirmed MM patients from the Hematology Department of the Affiliated Hospital of Nantong University (Nantong, China) between January 2013 and December 2018 were used in this study, with a median follow-up duration of 42 months from diagnosis. Patients with rheumatoid arthritis and other autoimmune diseases, central nervous system diseases, diabetes, various acute and chronic infectious diseases, other tumor diseases, and mental disorders were excluded from the study. The treatment response was evaluated when the newly diagnosed patients received 4 cycles of chemotherapy. Thirty healthy volunteers were recruited as controls.

Venous blood was collected in a serum separator tube containing clot activation additive and a barrier gel (Vacuette, Kresmunster, Austria) before or after bortezomib-based treatment. Samples were centrifuged at $4^{\circ} \mathrm{C}, 1500 \mathrm{~g}$ for $10 \mathrm{~min}$; the supernatants were further centrifuged at $4^{\circ} \mathrm{C}, 12000 \mathrm{~g}$ for $10 \mathrm{~min}$ and the serum was transferred to a RNase/DNase-free tube and stored at $-80^{\circ} \mathrm{C}$ until use.

Serum $\mathrm{k}$ and $\lambda$ light chain as well as serum IgA and IgG were quantitated using commercially available Beckman Coulter kits (IMMAGE Immunochemistry Systems) and the results were analyzed using Immage Special Protein Analyzer by immunoturbidimetry. Lactate dehydrogenase (LDH) was measured with Beckman Coulter Automatic Biochemical Analyzer using commercial Beckman Coulter kits by Lactic acid substrate method. Serum $\beta_{2}$ microglobulin was quantified with immunoturbidimetric test in Beckman Coulter Automatic Biochemical Analyzer using commercial Beckman Coulter kits.

BM samples were obtained from $12 \mathrm{MM}$ patients and 6 healthy controls for evaluation of miRNA expression levels in serum-BM paired samples. BM samples were collected by BM aspiration in tubes containing ethylenediaminetetraacetic acid (EDTA) before bortezomib treatment and CD138 + plasma cells were immediately isolated from BM by CD138 + magnetic bead separation technology following manufacturer's instructions (Miltenyi Biotec Corp., Gladbach, Germany). The purities of isolated CD138 + cells from MM patients and healthy donors were around $90 \%$ and $78 \%$, respectively. Written informed consent was obtained from all patients, and the study was approved by the Ethics Committee of our Hospital.

\section{RNA isolation and reverse transcription}

Total RNA including miRNAs was extracted from $300 \mu \mathrm{l}$ serum samples using the mirVana Paris RNA Isolation kit (Ambion, Austin, TX) following the manufacturer's protocol for liquid samples. Total RNA was isolated from CD138 + plasma cells using Trizol (Invitrogen, USA) reagent. The RNA concentration and purity were determined using a spectrophotometer (NanoPhotometerTM, IMPLEN, German). The serum RNA concentration ranged from $10 \mathrm{ng} / \mu \mathrm{l}$ to $20 \mathrm{ng} / \mu \mathrm{l}$ and RNA purity ranged from 1.20 to 1.86 at $260 / 280 \mathrm{~nm}, 0.05$ to 0.30 at $260 / 230 \mathrm{~nm}$, respectively. The range of isolated serum RNA amounts in total RNA was $0.30 \mu \mathrm{g}$ to $1.20 \mu \mathrm{g}$. 100ng RNA was reverse transcribed into complementary DNA (cDNA) using RevertAid First Strand cDNA Synthesis Kit (ThermoFisher Scientific, Waltham, USA) following the instructions.

\section{Real-time quantitative polymerase chain reaction (qRT-PCR) analysis}

qRT- PCR reactions were performed using ABI 7500 Real-Time PCR System ( $A B I$, Abilene, TX, USA) in triplicate. mRNA levels were qualified using Fast SYBR-Green Master Mix (Applied Biosystems Life Technologies) using U6 as the internal control. For detection of miR-148a and miR-152 expression levels, Bulge-loopTM miRNA qRT-PCR Primer Sets (one RT primer and a pair of qPCR primers for each set) specific for miR-148a and miR-152 designed by Ruibo Biotechnologies Co., Ltd (Guangzhou, China) were used, and endogenous $\mathrm{U} 6$ snRNA was used as control. All experimental results were calculated using $2^{-} \triangle \triangle \mathrm{CT}$ method: $\triangle \mathrm{Ct}=\mathrm{CT}$ (miR-148a and miR-152) - CT (U6), $\triangle \triangle C T=\triangle C T$ (patients) - $\triangle C T$ (control). The cutoff value of miR-148a and miR-152 was 1.345 and 0.62 , respectively. The PCR products were verified by sequencing. 


\section{Statistical analysis}

Statistical analysis was performed using SPSS Statistics 17.0 software (SPSS Inc., Chicago, IL, USA) and related graphs were drawn by GraphPad Prism v 5.0 software (GraphPad Software Inc, La Jolla, CA, USA). Kolmogorov-Smirnov test was used to determine the normality of data distribution in each group. Normally distributed variables are expressed as the mean \pm standard deviation (SD), and non-normally distributed variables are expressed as medians (25th, 75th percentiles). The relative expression of miR-148a and miR152 in MM and healthy control group was verified by M-W test. Correlation analysis was performed using Spearman tests. Associations of serum miR-148 or miR-152 with the clinicopathological parameters were examined by $\chi 2$ test. Kaplan-Meier survival curves were constructed and Cox regression model was applied to evaluate the prognostic value of each variable to overall survival (OS) of MM patients. Receiver-operating characteristic curves (ROC) and area under the ROC curve (AUC) were used to assess the diagnostic performance of miR-148a and miR-152 for MM. $\mathrm{P}<0.05$ was considered statistically significant.

\section{Results}

\section{Baseline participant characteristics}

The clinicopathologic characteristics of the 90 participants are listed in Table 1. No significant variation in age and sex distribution was observed between MM patients and healthy controls $(P>0.05)$. The median age of the $90 \mathrm{MM}$ patients and 30 healthy volunteers was $63(40-84)$ and 62 years (43-79) years respectively.

Table 1

Characteristics of the study subjects

\begin{tabular}{|c|c|c|c|}
\hline Characteristics & MM & Control & $\mathbf{P}$ \\
\hline No. of patients & 90 & 30 & \\
\hline Age & & & 0.68 \\
\hline Mean (range) & $63(40-84)$ & $62(43-79)$ & \\
\hline Gender & & & 0.92 \\
\hline Male & 56 & 19 & \\
\hline Female & 34 & 11 & \\
\hline $\lg G$ & $10.15(5.79,25.55)$ & $13.05(10.55,17.18)$ & 0.39 \\
\hline $\lg A$ & $0.92(0.36,15.23)$ & $2.00(1.37,3.91)$ & 0.071 \\
\hline$\lambda$ light chain & $354(197.30,1065)$ & $463.50(408.50,581)$ & 0.081 \\
\hline $\mathrm{K}$ light chain & $1320(481,2505)$ & $901.50(782.30,1065)$ & 0.23 \\
\hline LDH & $248(176.80,285)$ & $176.50(150.30,200.30)$ & $<0.0001$ \\
\hline$\beta_{2}$ microglobulin & $2.67(1.61,4.72)$ & $1.90(1.70,2.13)$ & 0.001 \\
\hline hemoglobin & $89.50 \pm 26.80$ & $138.30 \pm 13.40$ & $<0.0001$ \\
\hline erythrocyte sedimentation rate & $72.00 \pm 38.50$ & $6.40 \pm 3.20$ & $<0.0001$ \\
\hline creatinine & $100.50(58.80,151.30)$ & $70.00(62.00,84.75)$ & 0.0075 \\
\hline albumin & $31.20 \pm 5.50$ & $43.30 \pm 2.50$ & $<0.0001$ \\
\hline uric acid & $443.10 \pm 177.10$ & $318.60 \pm 55.50$ & 0.0001 \\
\hline urea & $9.60 \pm 4.00$ & $5.60 \pm 1.10$ & 0.92 \\
\hline
\end{tabular}

\section{Validation of miR-148a and miR-152 in CD138 + plasma cells}


It was found in our previous study that miR-148a and miR-152 were upregulated in CD138 + plasma cells of myeloma patients [20], which was further verified by RT-qPCR in this study $(P=0.02$ and $P=0.03$ respectively) (Fig. $1 \mathrm{a}, \mathrm{b})$. In addition, a significant positive correlation was observed between the relative expression of miR-148a $(r=0.68, P=0.02)$ and miR-152 $(r=0.60, P=0.04)$ in $C D 138^{+}$ plasma cells and serum (Fig. 1c, d).

\section{Validation of serum miR-148a and miR-152 expression levels}

The relative expression level of miR-148a and miR-152 in serum of MM patients was significantly higher than that in healthy controls (both $\mathrm{P}<0.05$ ) (Fig. 2a, b). In addition, the relative expression level of miR-148a and miR-152 showed a strong correlation with each other, with a correlation coefficient of $0.3346(\mathrm{P}<0.05)$ (Fig. 2c). The association of the serum miR-148a and miR-152 expression pattern with the treatment status was further analyzed. As shown in Fig. 3a, b, there was a significant difference in the expression level of miR-152 before and after treatment, whereas the level of miR-148a showed no such a significant difference ( $P=0.0059$ vs. $P$ $=0.27$ ). Analysis of serial changes of miR-148a and miR-152 expression in two MM patients who underwent various routine examinations during treatment and follow-up demonstrated that the expression of miR-148a and miR-152 changed dynamically and was associated with the personalized disease status (Fig. 3c, d). The PCR amplification products of miR-148a and miR-152 were further validated by sequencing. These objective gene sequences were in accordance with those provided by the National Center of Bioinformatics Institute (NCBI).

\section{Association of serum miR-148a and miR-152 expression with the clinicopathologic parameters}

Spearman bivariate analysis showed that the relative expression of miR-148a/miR-152 was significantly correlated with $\lg A+\lg G(r=$ $0.28, P=0.01 / r=0.26, P=0.01), K+\lambda$ light chain $(r=0.25, P=0.02 / r=0.24, P=0.02), \beta_{2}$ microglobulin $(r=0.26, P=0.01 / r=0.25, P=$ $0.02)$ and $\operatorname{LDH}(r=0.23, P=0.03 r=0.22, P=0.03)$ (Fig. 4a-d/Fig. 4e-h). MM patients were stratified by age, gender, international staging system, plasma cell percentage in BM, anemia, renal insufficiency, bone disease and treatment response. Based on the median level of miR-148a or miR-152, MM patients were categorized into a low-expression group and a high-expression group. The results showed that high serum expression of miR-152 and miR-148a was significantly associated with the international staging system $(P=0.02,0.0063)$, plasma cell percentage in $B M(P=0.034,0.01)$ and treatment response $(P=0.0011,0.0002)$, but not with the other clinicopathologic parameters (all $\mathrm{P}>0.05$, Table 2 ). 
Table 2

Correlations of serum miR-148a and miR-152 expressions with the clinicopathologic characteristics of MM patients

\begin{tabular}{|c|c|c|c|c|c|c|c|}
\hline \multirow[t]{2}{*}{ Characteristics } & \multirow[t]{2}{*}{$\mathrm{n}$} & \multicolumn{3}{|c|}{ miR-152 expression } & \multicolumn{3}{|c|}{ miR-148a expression } \\
\hline & & High & Low & $\mathbf{P}$ & High & Low & $\mathbf{P}$ \\
\hline \multicolumn{8}{|l|}{ Age (years) } \\
\hline$<60$ & 31 & 15 & 16 & 1.0 & 13 & 18 & 0.38 \\
\hline$\geq 60$ & 59 & 30 & 29 & & 32 & 27 & \\
\hline \multicolumn{8}{|l|}{ Gender } \\
\hline Male & 56 & 30 & 26 & 0.51 & 29 & 27 & 0.83 \\
\hline Female & 34 & 15 & 19 & & 16 & 18 & \\
\hline \multicolumn{8}{|c|}{ International staging system } \\
\hline Stage $1, \mathrm{n}(\%)$ & 29 & 9 & 20 & 0.023 & 8 & 21 & 0.0063 \\
\hline Stage $2+3, \mathrm{n}(\%)$ & 61 & 36 & 25 & & 37 & 24 & \\
\hline \multicolumn{8}{|c|}{ Percentage of plasma cell in bone marrow (\%) } \\
\hline$>10 \%$ & 41 & 26 & 15 & 0.034 & 27 & 14 & 0.011 \\
\hline$<10 \%$ & 49 & 19 & 30 & & 18 & 31 & \\
\hline \multicolumn{8}{|l|}{ Anemia, n (\%) } \\
\hline Negative & 34 & 16 & 18 & 0.83 & 13 & 21 & 0.13 \\
\hline Positive & 56 & 29 & 27 & & 32 & 24 & \\
\hline \multicolumn{8}{|c|}{ Renal insufficiency, n (\%) } \\
\hline Negative & 34 & 14 & 20 & 0.28 & 21 & 13 & 0.13 \\
\hline Positive & 56 & 31 & 25 & & 24 & 32 & \\
\hline \multicolumn{8}{|l|}{ Bone disease, $\mathrm{n}(\%)$} \\
\hline Negative & 41 & 19 & 22 & 0.67 & 18 & 23 & 0.40 \\
\hline Positive & 49 & 26 & 23 & & 27 & 22 & \\
\hline \multicolumn{8}{|l|}{ Treatment response } \\
\hline $\mathrm{CR}+\mathrm{VGPR}+\mathrm{PR}, \mathrm{n}(\%)$ & 63 & 24 & 39 & 0.0011 & 23 & 40 & 0.0002 \\
\hline $\mathrm{SD}+\mathrm{PD}, \mathrm{n}(\%)$ & 27 & 21 & 6 & & 22 & 5 & \\
\hline
\end{tabular}

\section{Diagnostic performance of serum miR-148a and miR-152}

ROC analysis was used to investigate the diagnostic value of both miRNAs. AUC of miR-148a was 0.75 ( $95 \%$ Cl: $0.66-0.84, p<$ 0.0001 ) with $58.89 \%$ sensitivity and $90 \%$ specificity. Serum miR-152 was slightly better in differentiating between MM patients and healthy controls with an AUC of 0.84 (95\% Cl: $0.77-0.92, p<0.0001)$, sensitivity of $72.22 \%$ and specificity of $86.67 \%$. Notably, multivariate logistical regression analysis suggested that the combination of miR-152 and miR-148a could improve the predictive value, offering an AUC value of 0.86 (95\% Cl: 0.79 to $0.93, \mathrm{P}<0.0001)$, a sensitivity of $77.78 \%$ and a specificity $93.33 \%$ for $\mathrm{MM}$, all of which were significantly higher than serum miR-148a or miR-152 alone (both $\mathrm{P}<0.05$ ) (Fig. 5a). Further ROC analysis showed that the combination of miR-148a, miR-152 and $\beta_{2}$ microglobulin had an increased AUC value to 0.94 (95\% Cl: 0.89 to 0.98 ) with a sensitivity of $90 \%$ and a specificity of $90 \%$ (Table 3 ). 
Table 3

Diagnosis performance of circulating miR-148a and miR-152 for MM

\begin{tabular}{|c|c|c|c|c|c|c|c|c|c|}
\hline & Accuracy & Sensitivity & Specificity & $\begin{array}{l}\text { Positive } \\
\text { predictive } \\
\text { value }\end{array}$ & $\begin{array}{l}\text { Negative } \\
\text { predictive } \\
\text { value }\end{array}$ & $\begin{array}{l}\text { Positive } \\
\text { likelihood } \\
\text { ratio }\end{array}$ & $\begin{array}{l}\text { Negative } \\
\text { likelihood } \\
\text { ratio }\end{array}$ & $\begin{array}{l}\text { Youden } \\
\text { index }\end{array}$ & $\begin{array}{l}\text { Cutoff } \\
\text { point }\end{array}$ \\
\hline miR-148a & $66.67 \%$ & $58.89 \%$ & $90 \%$ & $94.64 \%$ & $42.19 \%$ & $5.89 \%$ & $45.68 \%$ & $48.89 \%$ & 1.35 \\
\hline miR-152 & $75.83 \%$ & $72.22 \%$ & $86.67 \%$ & $94.20 \%$ & $50.98 \%$ & $5.42 \%$ & $32.05 \%$ & $58.89 \%$ & 0.62 \\
\hline $\begin{array}{l}\text { miR-148a + } \\
\text { miR-152 }\end{array}$ & $81.67 \%$ & $77.78 \%$ & $93.33 \%$ & $97.22 \%$ & $58.33 \%$ & $11.66 \%$ & $23.81 \%$ & $71.11 \%$ & 0.75 \\
\hline $\begin{array}{l}\beta_{2} \\
\text { microglobulin }\end{array}$ & $65 \%$ & $54.44 \%$ & $96.67 \%$ & $98 \%$ & $41.43 \%$ & $16.33 \%$ & $47.13 \%$ & $51.11 \%$ & 2.52 \\
\hline $\begin{array}{l}\operatorname{miR}-148 a+ \\
\text { miR-152+ } \\
\beta_{2} \text { microglobulin }\end{array}$ & $90 \%$ & $90 \%$ & $90 \%$ & $96.43 \%$ & $75 \%$ & $9 \%$ & $11.11 \%$ & $80 \%$ & 0.59 \\
\hline
\end{tabular}

\section{Prognostic prediction of serum miR-148a and miR-152 in MM patients}

The median follow-up duration was 42 (34-60) months. Kaplan-Meier survival analysis revealed that OS in MM patients with high miR-148a and miR-152 expression levels was significantly reduced as compared with that in those with low miR-148a $(P=0.024$, Fig. $5 b)$ and miR-152 expression levels $(P=0.027$, Fig. $5 c)$. As shown in Fig. $5 d$, OS was low in MM patients with both miR-148a and miR152 high expressions as compared with that in patients with other combinations of high or low levels of these miRNAs. Parameters significantly related to OS in the univariate analysis were subjected to multivariate analysis to identify independent factors for prognosis and the results showed that miR-148a level $(P=0.034)$ and miR-152 level $(P=0.039)$ were independent prognostic factors for OS in MM with their combined effect being the strongest predictor $(P=0.008)$ (Table 4).

Table 4

Univariate and multivariate Cox proportional hazards regression model analysis of overall survival in MM patients

\begin{tabular}{|c|c|c|c|c|c|}
\hline \multirow[t]{2}{*}{ Parameters } & \multirow[t]{2}{*}{ Categories } & \multicolumn{2}{|l|}{ Univariate analysis } & \multicolumn{2}{|l|}{ Multivariate analysis } \\
\hline & & $\mathrm{HR}(95 \% \mathrm{Cl})$ & $\mathbf{P}$ & $\mathrm{HR}(95 \% \mathrm{Cl})$ & $\mathbf{P}$ \\
\hline Age & $<60$ vs. $\geq 60$ & $2.38(1.011-5.60)$ & 0.047 & & 0.053 \\
\hline Sex & Male vs. female & $2.16(0.85-5.48)$ & 0.11 & & \\
\hline miR-148a & Low vs. high & $2.69(1.14-6.35)$ & 0.024 & $2.96(1.08-8.06)$ & 0.034 \\
\hline miR-152 & Low vs. high & $2.70(1.12-6.50)$ & 0.027 & $3.18(1.06-9.55)$ & 0.039 \\
\hline$m i R-148 a+m i R-152$ & Low vs. high & $3.56(1.51-8.38)$ & 0.0036 & $3.92(1.43-10.75)$ & 0.0080 \\
\hline $\begin{array}{l}\text { International staging } \\
\text { system }\end{array}$ & Stage 1 vs. Stage $2+3$ & $0.35(0.14-0.83)$ & 0.017 & $3.82(1.13-12.88)$ & 0.031 \\
\hline Treatment response & $\begin{array}{l}C R+V G P R+P R \text { vs. SD + } \\
P D\end{array}$ & $0.069(0.029-0.16)$ & $<0.0001$ & $114.70(3.50-3790.10)$ & 0.0080 \\
\hline
\end{tabular}

\section{Discussion}

The current study on the miR-148/152 family in MM revealed that the expression of miR-148a and miR-152 was significantly higher in the serum \& CD138 + plasma cells of MM patients as compared with healthy controls. There was significant difference in miR-152 expression before and after treatment. The expression of miR-148a and miR-152 was positively correlated with the clinicopathological factors, as well as with the international staging system, plasma cell percentage in BM and treatment response.

Some studies reported that miR-148a was downregulated in gastric, non-small cell lung cancer and cervical cells [21-23], while others reported that it was upregulated [24-26]. Our results demonstrated that miR-148a was significantly upregulated in both serum and plasma cells of MM patients, which is consistent with the report that serum miR-148a-3p level was increased in prostate cancer specimens as compared with normal controls [27]. In addition, miR-148a was also shown to be up-regulated in serum samples from 
Type 1 diabetes patients $v s$. non-diabetic controls [28]. In contrast, Peng et al [29] observed downregulation of miR-148a in the serum of patients with colorectal cancer when compared with healthy controls. It was previously reported that miR-152 acted as a tumor suppressor and it was lowly expressed in cervical cancer tissues and cell lines [30-32]. Contrary to other studies [33-35], we found that miR-152 was upregulated in both the serum and plasma cells of MM patients. Consistent with our research, Jiang et al [36] also observed an upregulation of miR-152 in the serum of patients with bladder cancer, and that miR-152 was an independent prognosis factor for tumor recurrence in patients with non-muscle invasive bladder cancer (NMIBC). Similarly, miR-152 was reported to be upregulated in BM and cells of patients with chronic myeloid leukemia compared with healthy controls. Further studies showed that miR-152 promoted the proliferation of K562 cells and inhibited cell apoptosis [37]. Another study revealed that miR-152 plasma levels were increased in diabetic nephropathy cases compared with normal controls [38]. However, a downregulation of serum miR-152 was detected in patients with uterine sarcoma [39]. We think that this discrepancy is likely due to the fact that tumor micro-environment varies in different types of cancers and therefore the roles of miRNAs, their target genes and relative mechanisms may be different depending on the type and location of cancer. However, the mechanistic insights of miR-148/miR-152 regulation in carcinogenesis and their significant contributions to tumor progression need to be further investigated in different types of cancer. Notably, we found that the expression of miR-148a and miR-152 changed dynamically and was associated with the personalized disease status, which may aid in improving treatment outcomes by monitoring the patients for these parameters.

Quantitative determination of immunoglobulins, $L D H, \beta_{2}$ microglobulin, determination of free light chains (including free light chain ratio) are considered a standard diagnostic approach for MM [2]. In our study, AUC was 0.75 and 0.84 for serum miR-148a and miR152 respectively, while AUC was slightly increased to 0.86 when the two miRNAs were combined. We also observed a statistically significant correlation of serum miR-148a or miR-152 expression with LDH, IgA $+\lg G, \beta_{2}$ microglobulin and $k+\lambda$ light chain. In addition, higher miR-148a and miR-152 expression levels respectively correlated with shorter OS of MM patients. Interestingly, miR148a level, miR-152 level, international staging system and treatment response were independent prognostic factors for OS of MM patients. Higher levels of these two miRNAs were also correlated with shorter OS of MM patients. These findings suggest that a combination of these two miRNAs might be better for identifying the pathological stage and predicting the prognosis of MM patients more accurately than detection of a single miRNA.

Several limitations are recognized in our study. Firstly, it is unknown whether miR-148a and miR-152 are capable of distinguishing newly diagnosed MM from others such as MGUS, smoldering MM, relapsed and refractory MM. Secondly, the sample size is relatively small, so further studies and validation with larger cohorts including long-term clinical data with well-defined clinical staging and outcomes are needed to confirm our conclusion. Thirdly, the biological functions of the miR-148/152 family were not investigated, and hence their exact roles in the development and progression of MM remain to be further clarified in future.

In summary, this is the first report describing the aberrant expression of the miR-148/152 family in both CD $138+$ plasma cells and serum of MM patients. It was found that the expression of miR-148a and miR-152 in the serum of MM patients was upregulated with high sensitivity and specificity. Notably, the combination of miR-148a and miR-152 could improve the diagnostic and predictive value. A high level of serum miR-148a and miR-152 was associated with tumor progression and poor clinical outcomes of MM. These findings may lay a foundation for the development of novel noninvasive tests to diagnose and predict the prognosis of MM and pave the way for further study of innovative therapeutic strategies in clinical practice for MM.

\section{Declarations}

\section{Acknowledgements}

The authors received no financial support for the research, authorship, and/or publication of this article.

\section{Declaration of Conflicting Interests}

The authors declare that there is no conflict of interest.

\section{Statement of Ethics}


Written informed consent was obtained from all patients, and the study was approved by the Ethics Committee of the Affiliated Hospital of Nantong University (2012-005).

\section{Author Contributions}

Lingling Xie contributed to designing and drafting the manuscript. Guangfei Xie, Xiuying Shi and Hongming Huang were responsible for analysis. Shaoqing Ju and Xudong Wang critically revised the manuscript. All the authors gave final approval of this article.

\section{References}

[1] M. Kehrer, S. Koob, A. Strauss, D.C. Wirtz, J. Schmolders, [Multiple Myeloma - Current Status in Diagnostic Testing and Therapy], Z. Orthop. Unfall. 155(5) (2017) 575-586.

[2] C. Gerecke, S. Fuhrmann, S. Strifler, M. Schmidt-Hieber, H. Einsele, S. Knop, The Diagnosis and Treatment of Multiple Myeloma, Dtsch Arztebl Int 113(27-28) (2016) 470-6.

[3] H. Zhang, X. Huang, L. Ye, G. Guo, X. Li, C. Chen, L. Sun, B. Li, N. Chen, X. Xue, B Cell-Related Circulating MicroRNAs With the Potential Value of Biomarkers in the Differential Diagnosis, and Distinguishment Between the Disease Activity and Lupus Nephritis for Systemic Lupus Erythematosus, Front. Immunol. 9 (2018) 1473.

[4] C. Papadaki, M. Stratigos, G. Markakis, M. Spiliotaki, G. Mastrostamatis, C. Nikolaou, D. Mavroudis, S. Agelaki, Circulating microRNAs in the early prediction of disease recurrence in primary breast cancer, Breast Cancer Res. 20(1) (2018) 72.

[5] A. Tölle, C.C. Blobel, K. Jung, Circulating miRNAs in blood and urine as diagnostic and prognostic biomarkers for bladder cancer: an update in 2017, Biomark. Med. 12(6) (2018) 667-676.

[6] A. Allegra, A. Alonci, S. Campo, G. Penna, A. Petrungaro, D. Gerace, C. Musolino, Circulating microRNAs: new biomarkers in diagnosis, prognosis and treatment of cancer (review), Int. J. Oncol. 41(6) (2012) 1897-912.

[7] B. Dewdney, A. Trollope, J. Moxon, D. Thomas Manapurathe, E. Biros, J. Golledge, Circulating MicroRNAs as Biomarkers for Acute Ischemic Stroke: A Systematic Review, J. Stroke Cerebrovasc. Dis. 27(3) (2018) 522-530.

[8] P.S. Mitchell, R.K. Parkin, E.M. Kroh, B.R. Fritz, S.K. Wyman, E.L. Pogosova-Agadjanyan, A. Peterson, J. Noteboom, K.C. O'Briant, A. Allen, D.W. Lin, N. Urban, C.W. Drescher, B.S. Knudsen, D.L. Stirewalt, R. Gentleman, R.L. Vessella, P.S. Nelson, D.B. Martin, M. Tewari, Circulating microRNAs as stable blood-based markers for cancer detection, Proc. Natl. Acad. Sci. U. S. A. 105(30) (2008) $10513-8$.

[9] Q. Li, L. Liu, W. Li, Identification of circulating microRNAs as biomarkers in diagnosis of hematologic cancers: a meta-analysis, Tumour Biol. 35(10) (2014) 10467-78.

[10] X. Qu, M. Zhao, S. Wu, W. Yu, J. Xu, J. Xu, J. Li, L. Chen, Circulating microRNA 483-5p as a novel biomarker for diagnosis survival prediction in multiple myeloma, Med. Oncol. 31(10) (2014) 219.

[11] L. Kubiczkova, F. Kryukov, O. Slaby, E. Dementyeva, J. Jarkovsky, J. Nekvindova, L. Radova, H. Greslikova, P. Kuglik, E. Vetesnikova, L. Pour, Z. Adam, S. Sevcikova, R. Hajek, Circulating serum microRNAs as novel diagnostic and prognostic biomarkers for multiple myeloma and monoclonal gammopathy of undetermined significance, Haematologica 99(3) (2014) 511-8.

[12] S.H. Jung, S.E. Lee, M. Lee, S.H. Kim, S.H. Yim, T.W. Kim, C.K. Min, Y.J. Chung, Circulating microRNA expressions can predict the outcome of lenalidomide plus low-dose dexamethasone treatment in patients with refractory/relapsed multiple myeloma, Haematologica 102(11) (2017) e456-e459.

[13] L. Besse, L. Sedlarikova, F. Kryukov, J. Nekvindova, L. Radova, O. Slaby, P. Kuglik, M. Almasi, M. Penka, M. Krejci, Z. Adam, L. Pour, S. Sevcikova, R. Hajek, Circulating Serum MicroRNA-130a as a Novel Putative Marker of Extramedullary Myeloma, PLoS One 10(9) (2015) e0137294. 
[14] C.I. Jones, M.V. Zabolotskaya, A.J. King, H.J. Stewart, G.A. Horne, T.J. Chevassut, S.F. Newbury, Identification of circulating microRNAs as diagnostic biomarkers for use in multiple myeloma, Br. J. Cancer 107(12) (2012) 1987-96.

[15] J. Chi, E. Ballabio, X.H. Chen, R. Kušec, S. Taylor, D. Hay, D. Tramonti, N.J. Saunders, T. Littlewood, F. Pezzella, J. Boultwood, J.S. Wainscoat, C.S. Hatton, C.H. Lawrie, MicroRNA expression in multiple myeloma is associated with genetic subtype, isotype and survival, Biol. Direct 6 (2011) 23.

[16] W. Wang, M. Corrigan-Cummins, E.A. Barber, L.M. Saleh, A. Zingone, A. Ghafoor, R. Costello, Y. Zhang, R.J. Kurlander, N. Korde, A.M. Roccaro, I.M. Ghobrial, O. Landgren, K.R. Calvo, Aberrant Levels of miRNAs in Bone Marrow Microenvironment and Peripheral Blood of Myeloma Patients and Disease Progression, J. Mol. Diagn. 17(6) (2015) 669-78.

[17] I.P.N. Bong, C.C. Ng, P. Baharuddin, Z. Zakaria, MicroRNA expression patterns and target prediction in multiple myeloma development and malignancy, Genes Genomics 39(5) (2017) 533-540.

[18] Y. Li, X. Deng, X. Zeng, X. Peng, The Role of Mir-148a in Cancer, J. Cancer 7(10) (2016) 1233-41.

[19] X. Liu, J. Li, F. Qin, S. Dai, miR-152 as a tumor suppressor microRNA: Target recognition and regulation in cancer, Oncol. Lett. 11(6) (2016) 3911-3916.

[20] J. Yuan, Z. Su, W. Gu, X. Shen, Q. Zhao, L. Shi, C. Jin, X. Wang, H. Cong, S. Ju, MiR-19b and miR-20a suppress apoptosis, promote proliferation and induce tumorigenicity of multiple myeloma cells by targeting PTEN, Cancer Biomark. 24(3) (2019) 279-289.

[21] S. Kumar, S.K. Sharawat, A. Ali, V. Gaur, P.S. Malik, S. Kumar, A. Mohan, R. Guleria, Identification of differentially expressed circulating serum microRNA for the diagnosis and prognosis of Indian non-small cell lung cancer patients, Curr. Probl. Cancer 44(4) (2020) 100540.

[22] W. Zhang, Y. Li, miR-148a downregulates the expression of transforming growth factor- $\beta 2$ and SMAD2 in gastric cancer, Int. J. Oncol. 48(5) (2016) 1877-85.

[23] M.S. Han, J.M. Lee, S.N. Kim, J.H. Kim, H.S. Kim, Human Papillomavirus 16 Oncoproteins Downregulate the Expression of miR148a-3p, miR-190a-5p, and miR-199b-5p in Cervical Cancer, Biomed Res Int 2018 (2018) 1942867.

[24] H. Zhang, Y. Wang, T. Xu, C. Li, J. Wu, Q. He, G. Wang, C. Ding, K. Liu, H. Tang, F. Ji, Increased expression of microRNA-148a in osteosarcoma promotes cancer cell growth by targeting PTEN, Oncol. Lett. 12(5) (2016) 3208-3214.

[25] T. Lang, Y. Nie, MiR-148a participates in the growth of RPMI8226 multiple myeloma cells by regulating CDKN1B, Biomed. Pharmacother. 84 (2016) 1967-1971.

[26] I.A. Paunescu, R. Bardan, A. Marcu, D. Nitusca, A. Dema, S. Negru, O. Balacescu, L. Balacescu, A. Cumpanas, I.O. Sirbu, B. Petrut, E. Seclaman, C. Marian, Biomarker Potential of Plasma MicroRNA-150-5p in Prostate Cancer, Medicina (Kaunas) 55(9) (2019).

[27] S.A. Dybos, A. Flatberg, J. Halgunset, T. Viset, T. Rolfseng, S. Kvam, H. Skogseth, Increased levels of serum miR-148a-3p are associated with prostate cancer, APMIS 126(9) (2018) 722-731.

[28] G.E. Grieco, D. Cataldo, E. Ceccarelli, L. Nigi, G. Catalano, N. Brusco, F. Mancarella, G. Ventriglia, C. Fondelli, E. Guarino, I. Crisci, G. Sebastiani, F. Dotta, Serum Levels of miR-148a and miR-21-5p Are Increased in Type 1 Diabetic Patients and Correlated with Markers of Bone Strength and Metabolism, Noncoding RNA 4(4) (2018).

[29] X. Peng, J. Wang, C. Zhang, K. Liu, L. Zhao, X. Chen, G. Huang, Y. Lai, A three-miRNA panel in serum as a noninvasive biomarker for colorectal cancer detection, Int. J. Biol. Markers 35(3) (2020) 74-82.

[30] B. Li, Z. Xie, B. Li, miR-152 functions as a tumor suppressor in colorectal cancer by targeting PIK3R3, Tumour Biol. 37(8) (2016) 10075-84.

[31] F. Wang, H. Ying, B. He, Y. Pan, H. Sun, S. Wang, Circulating miR-148/152 family as potential biomarkers in hepatocellular carcinoma, Tumour Biol. 37(4) (2016) 4945-53. 
[32] H. Zhang, Y. Lu, S. Wang, X. Sheng, S. Zhang, MicroRNA-152 Acts as a Tumor Suppressor MicroRNA by Inhibiting Krüppel-Like Factor 5 in Human Cervical Cancer, Oncol. Res. 27(3) (2019) 335-340.

[33] H. Chen, H. Liu, H. Zou, R. Chen, Y. Dou, S. Sheng, S. Dai, J. Ai, J. Melson, R.A. Kittles, M. Pirooznia, M.J. Liptay, J.A. Borgia, Y. Deng, Evaluation of Plasma miR-21 and miR-152 as Diagnostic Biomarkers for Common Types of Human Cancers, J. Cancer 7(5) (2016) 490-9.

[34] F. Matin, V. Jeet, L. Moya, L.A. Selth, S. Chambers, J.A. Clements, J. Batra, A Plasma Biomarker Panel of Four MicroRNAs for the Diagnosis of Prostate Cancer, Sci. Rep. 8(1) (2018) 6653.

[35] P. Zarecki, M. Hackl, J. Grillari, M. Debono, R. Eastell, Serum microRNAs as novel biomarkers for osteoporotic vertebral fractures, Bone 130 (2020) 115105.

[36] X. Jiang, L. Du, L. Wang, J. Li, Y. Liu, G. Zheng, A. Qu, X. Zhang, H. Pan, Y. Yang, C. Wang, Serum microRNA expression signatures identified from genome-wide microRNA profiling serve as novel noninvasive biomarkers for diagnosis and recurrence of bladder cancer, Int. J. Cancer 136(4) (2015) 854-62.

[37] L. Wang, Y. Wang, J. Lin, MiR-152-3p promotes the development of chronic myeloid leukemia by inhibiting p27, Eur. Rev. Med. Pharmacol. Sci. 22(24) (2018) 8789-8796.

[38] M. Roux, C. Perret, E. Feigerlova, B. Mohand Oumoussa, P.J. Saulnier, C. Proust, D.A. Trégouët, S. Hadjadj, Plasma levels of hsamiR-152-3p are associated with diabetic nephropathy in patients with type 2 diabetes, Nephrol. Dial. Transplant. 33(12) (2018) 22012207.

[39] X. Tong, X. Wang, C. Wang, L. Li, Elevated levels of serum MiR-152 and miR-24 in uterine sarcoma: potential for inducing autophagy via SIRT1 and deacetylated LC3, Br. J. Biomed. Sci. 75(1) (2018) 7-12.

\section{Figures}



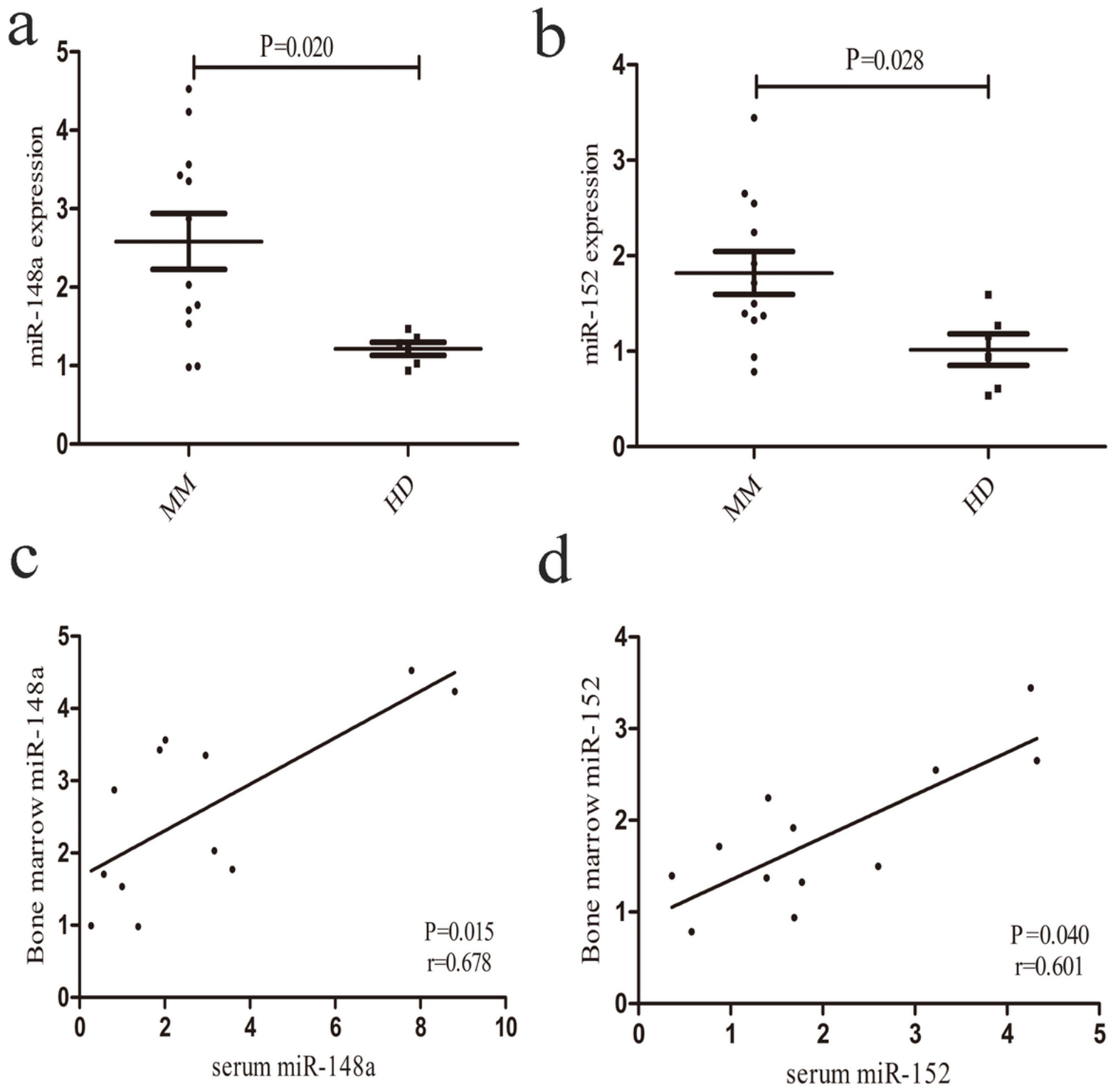

Figure 1

RT-qPCR analysis of the relative expression of miR-148a(a) and miR-152(b) in CD138+ plasma cells of 12 MM patients and 6 healthy controls. The correlations analysis with regard to the expression of miR-148a(c) and miR-152(d) between plasma cells and serum samples were analyzed in 12 paired samples of MM patients. $n=12$.
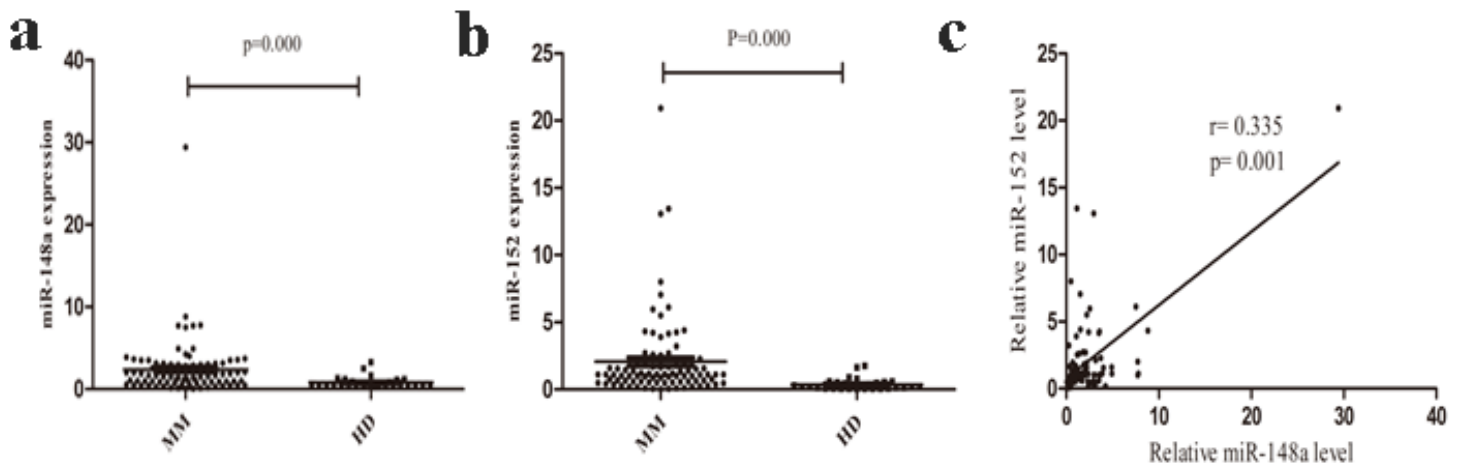

Figure 2 
qRT-PCR analysis of the relative expression level of miR-148a(a) and miR-152(b) in the serum of MM patients. Correlation of the expression level of circulating miR-148a vs. miR-152(c) in MM patients was analyzed. $n=90$.

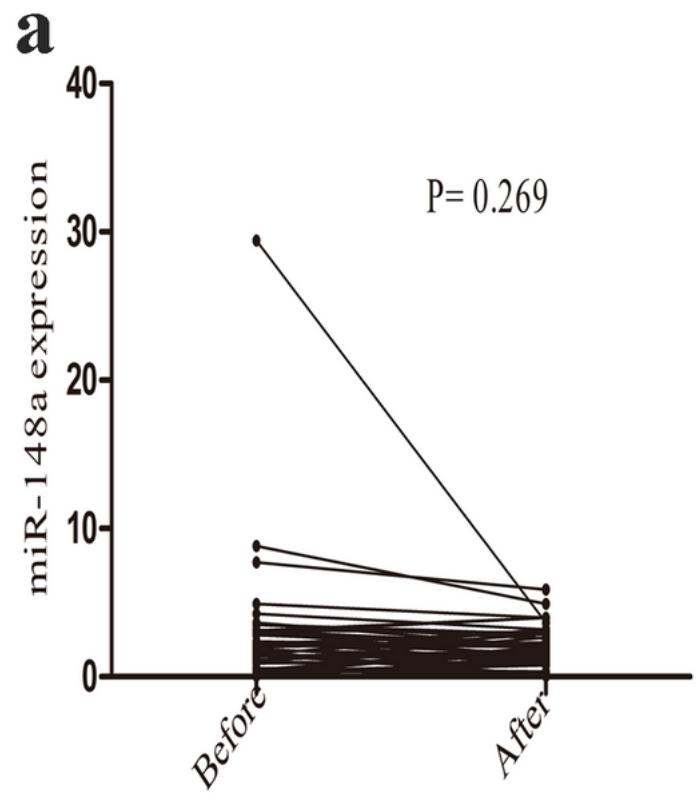

b
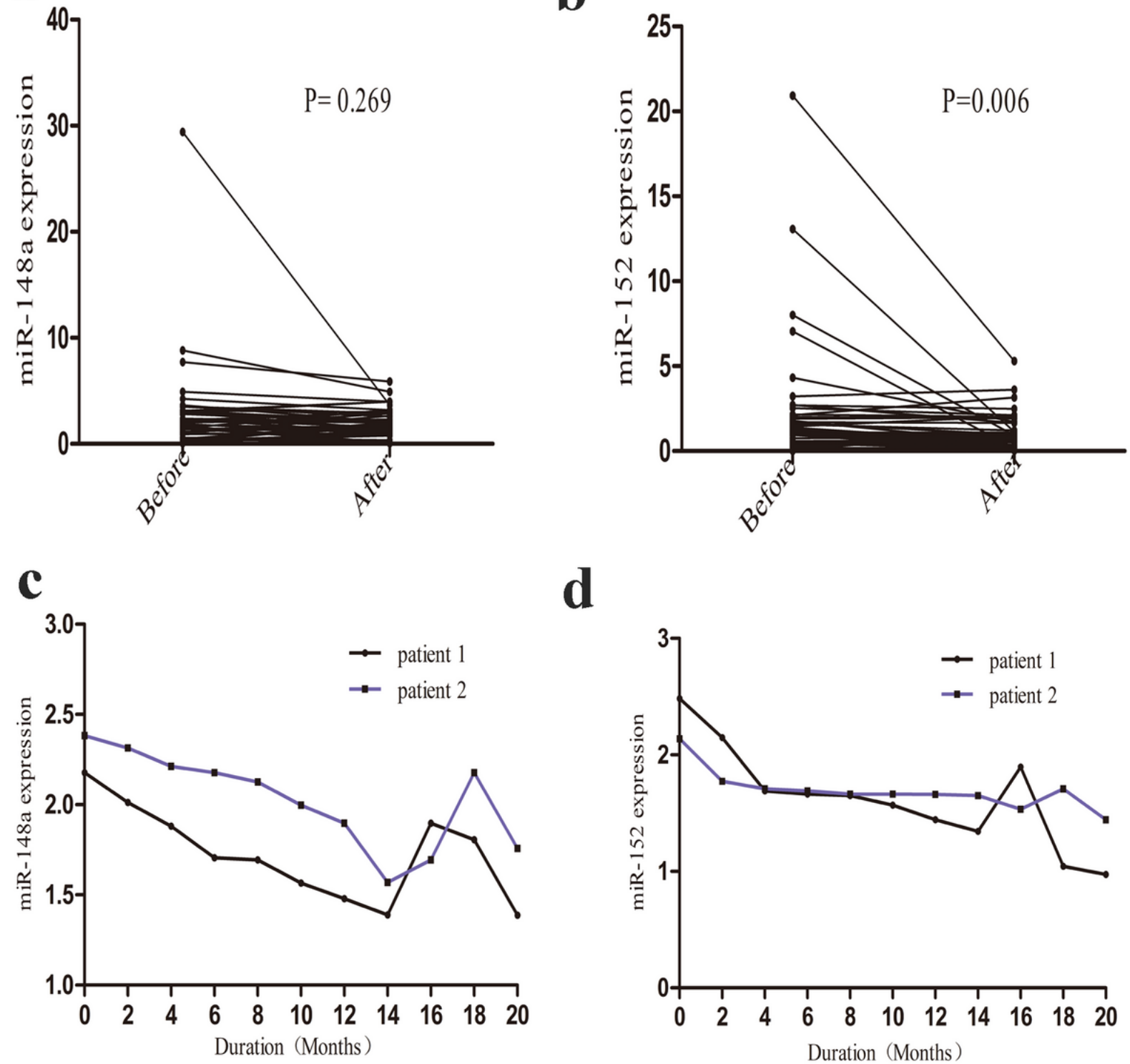

d

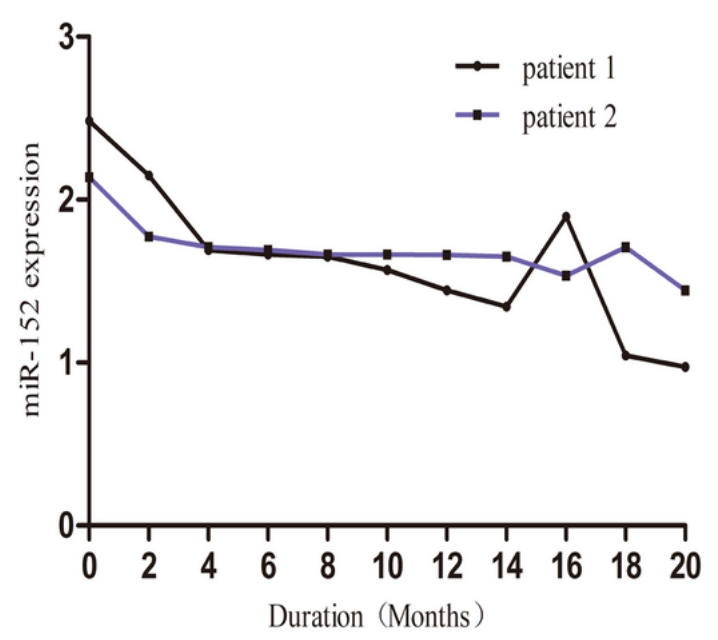

Figure 3

Line charts representing serum miR-148a(a) and miR-152(b) in MM patients before and after treatment. Expression of serum miR$148 \mathrm{a}(\mathrm{c})$ and miR-152(d) during treatment and follow-up in two representative patients. $\mathrm{n}=50$. 

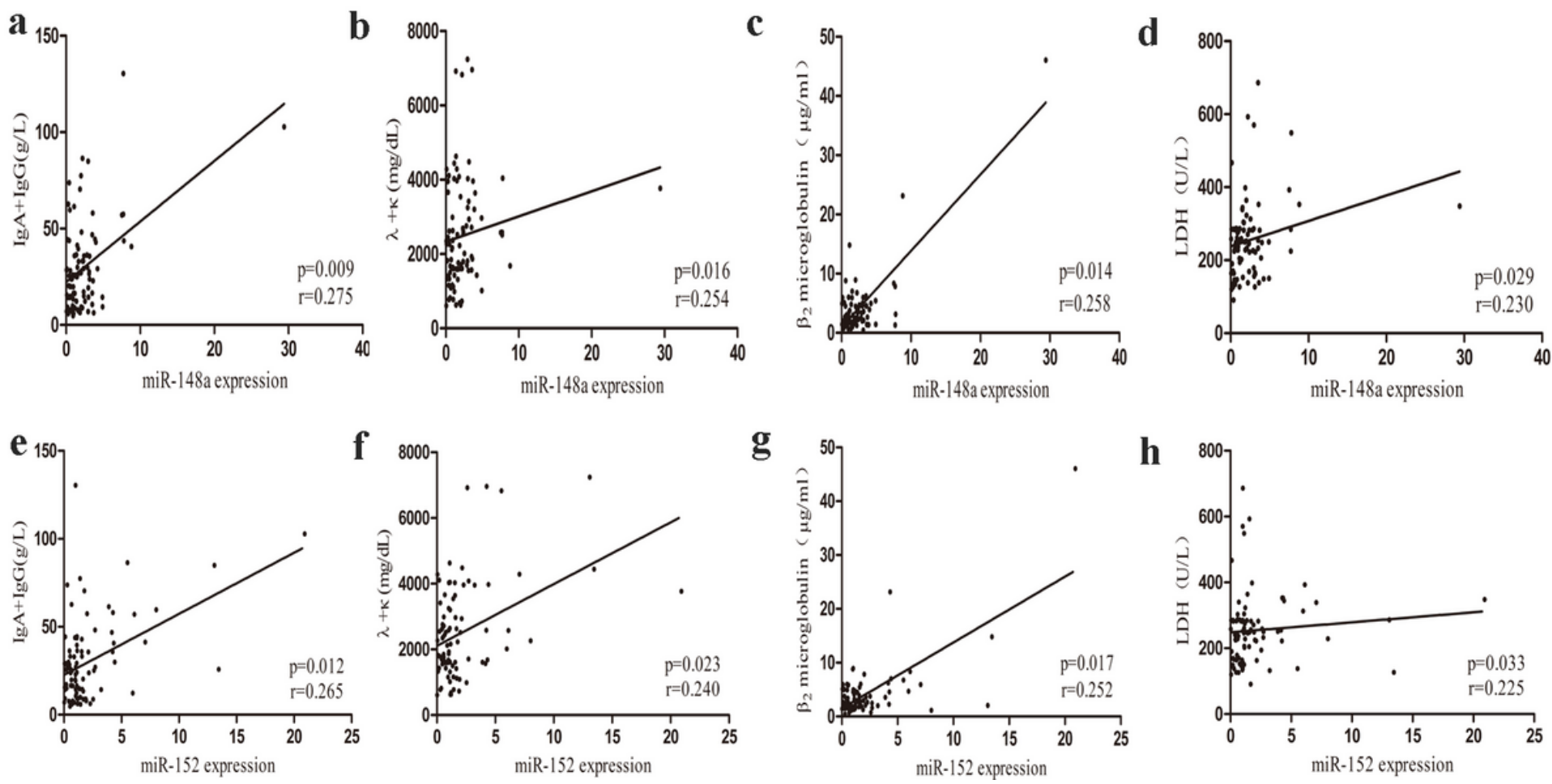

\section{Figure 4}

Correlations of serum miR-148a level with $\lg A+\operatorname{lgG}(a)$, $\mathrm{k}$ light chain $+\lambda$ light chain (b), $\beta 2$ microglobulin(c), $L D H(d)$. Correlations of serum miR-152 level with IgA + IgG(e), $k$ light chain $+\lambda$ light chain(f), $\beta 2$ microglobulin(g), LDH (h). $n=90$. 


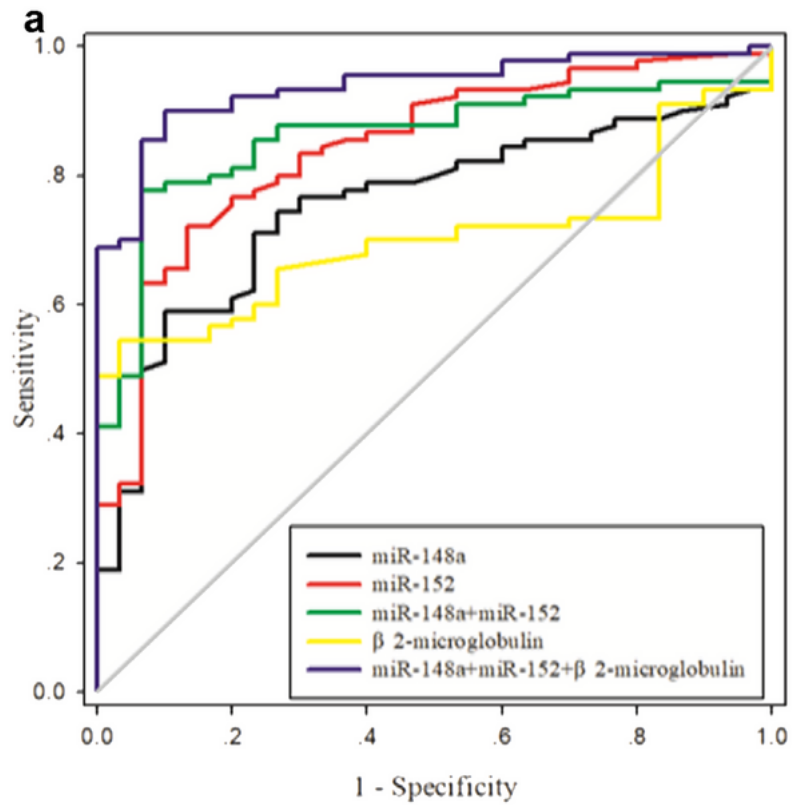

C

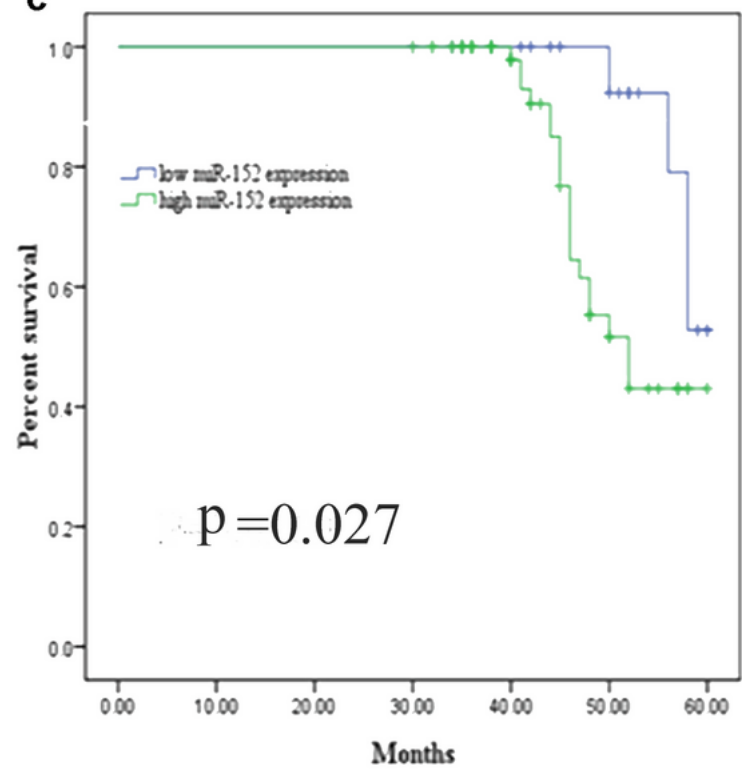

b

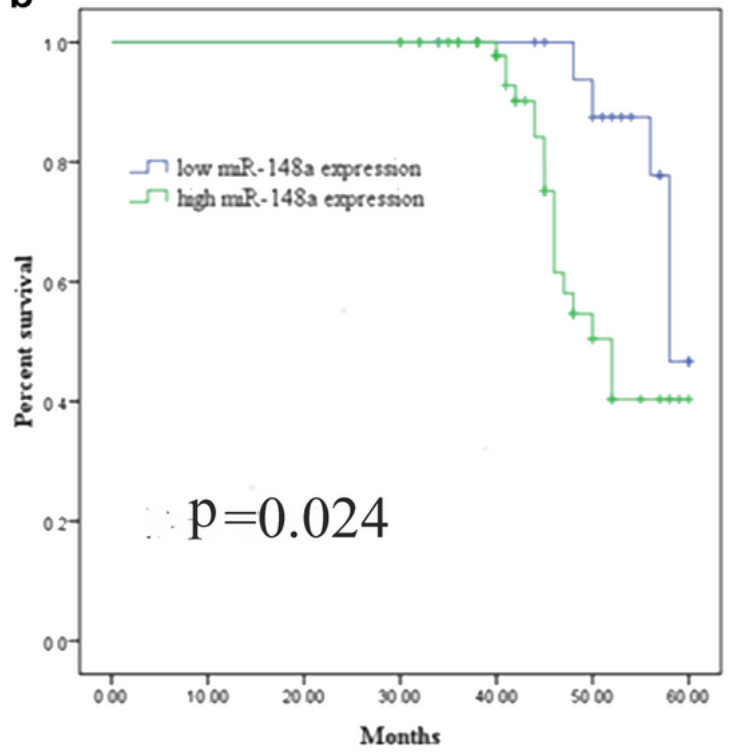

d

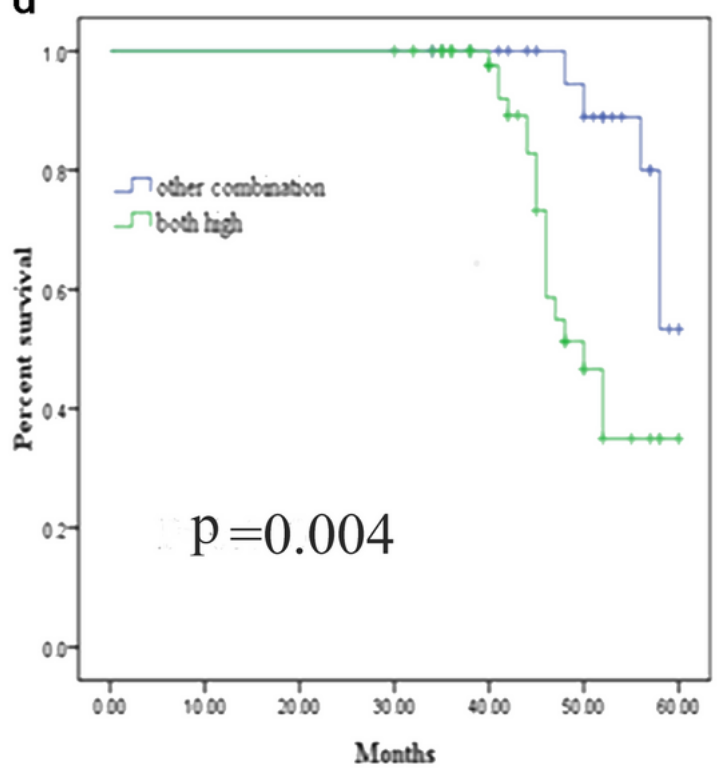

Figure 5

The diagnostic value of circulating miR-148a and miR-152 for MM(a). Kaplan-Meier curves for overall survival of MM patients were drawn according to the levels of serum miR-148a (b), miR-152(c) and combined miRNAs(d). $n=90$. 